\title{
"Quantity-effect" research strategy for comparison of antioxidant activity and quality of Rehmanniae Radix and Rehmannia Radix Praeparata by on-line HPLC-UV-ABTS assay
}

Hong-Ying $\mathrm{Li}^{1 \dagger}$, Jiang-Ji Fang ${ }^{2 \dagger}$, Hua-Dan Shen ${ }^{3}$, Xue-Qiong Zhang ${ }^{2}$, Xiao-Ping Ding ${ }^{1 *}$ and Jun-Feng Liu ${ }^{3 *}$

\begin{abstract}
Background: Quantitation analysis and chromatographic fingerprint of multi-components are frequently used to evaluate quality of herbal medicines but fail to reveal activity of the components. It is necessary to develop a rational approach of chromatography coupled with activity detection for quality assessment of herbal medicines.

Methods: An on-line HPLC-ultraviolet detection-2,2'-azino-bis(3-ethylbenzothiazoline-6-sulfonic acid) free radical scavenging (HPLC-UV-ABTS) method was developed to obtain the chromatographic fingerprints and ABTS ${ }^{+*}$ inhibition profiles (active fingerprints) of Rehmanniae Radix (Dihuang) and Rehmannia Radix Praeparata (Shu Dihuang). Eighteen compounds showing $\mathrm{ABTS}^{+*}$ inhibition activity were identified by HPLC-fourier-transform mass spectrometry (HPLC-FTMS). Verbascoside was used as a positive control to evaluate the total activities of the samples and the contribution rate of each compound. The similarities of the chromatographic and active fingerprints were estimated by the vectorial angle cosine method.

Results: The results showed that the HPLC-UV-ABTS method could efficiently detect antioxidant activity of the herbal medicine samples. The antioxidants were different between the two herbs and several new antioxidants were identified in Shu Dihuang. A function equation was generated in terms of the negative peak area $(x)$ and the concentrations of verbascoside $(y, \mu g / m L), y=2 E-07 x^{4}-8 E-05 x^{3}+0.0079 x^{2}+0.5755 x+1.4754, R^{2}=1$. Iridoid glycosides were identified as main antioxidants and showed their higher contributions to the total activity of the samples. The total contributions of the three main active components in the Dihuang and Shu Dihuang samples to the total activity, such as echinacoside, verbascoside and an unknown compound, were $39.2-58.1 \%$ and 55.9-69.4\%, respectively. The potencies of the main active components in the Shu Dihuang samples were two to ten times those in the Dihuang samples. Similarity values for S12 in the chromatographic fingerprints and S03, S12 and P03 in the active fingerprints were less than 0.9. The three batches of samples might show their different quality with the other samples.
\end{abstract}

Conclusions: The results suggested that the combination of "quantity-effect" research strategy and the HPLC-UV-ABTS analysis method could comprehensively evaluate the active components and quality of Dihuang and Shu Dhuang.

Keywords: HPLC-UV-ABTS, Rehmanniae Radix, Rehmannia Radix Praeparata, Quality evaluation, HPLC-FTMS

\footnotetext{
*Correspondence: dxp2888@126.com; liujf456@hotmail.com

${ }^{+}$Hong-Ying Li and Jiang-Ji Fang are contributed equally to this work and

should be considered co-first authors.

'Hubei Institute for Drug Control, Wuhan 430075, Hubei, China

${ }^{3}$ MOE Key Laboratory of Chinese Medicine Resource and Compound

Prescription, Hubei University of Chinese Medicine, Wuhan 430065, Hubei,

China

Full list of author information is available at the end of the article
}

(c) The Author(s). 2020 Open Access This article is distributed under the terms of the Creative Commons Attribution 4.0 International License (http://creativecommons.org/licenses/by/4.0/), which permits unrestricted use, distribution, and reproduction in any medium, provided you give appropriate credit to the original author(s) and the source, provide a link to the Creative Commons license, and indicate if changes were made. The Creative Commons Public Domain Dedication waiver (http://creativecommons.org/publicdomain/zero/1.0/) applies to the data made available in this article, unless otherwise stated. 


\section{Background}

Rehmannia glutinosa Libosch, one of the most common and important medicinal plants in China, is recorded in Chinese medical classic 'Shennong's Herba'. R. glutinosa based on the different processing methods is classified into three categories, namely fresh roots, dried roots (Dihuang), and steamed roots (Shu Dihuang), and they are applied to treat different diseases clinically. Shu Dihuang is usually applied to in the traditional Chinese medicine formulas that can nourish the Yin deficiency of liver, kidney and heart [1-3]. Many clinical and experimental studies have reported that the root of $R$. glutinosa possesses hypoglycaemic [4], anti-oxidant $[5,6]$ anti-cancer [7], anti-inflammatory [8], and immuneenhancement effects [9].

Iridoid glycosides are considered the main active ingredients in Dihuang. At present, a number of chemical constituents from Dihuang, such as iridoids, ionone glucosides, sesquiterpenes and phenylethanoid glycosides [10-12], have been isolated and identified. In the Chinese Pharmacopoeia (Ch. P), catalpol and verbascoside were quantified by HPLC methods to control the quality of Dihuang and Shu Dihuang, and 1,1-Diphenyl-2-picrylhydrazyl (DPPH) scavenging method was applied to distinguish verbascoside through thin layer chromatography (TLC) [13]. However, these methods might not be able to reveal efficiently the bioactivity of the compounds in Dihuang and Shu Dihuang.

Free radicals may be involved in cancer, ageing and cardiovascular diseases [14]. There has been a large research focus on antioxidants, especially plant-derived antioxidants. In order to identify efficiently antioxidants in complex extracts, the combinational techniques such as HPLC-DAD-DPPH, HPLC-DAD-ABTS, DPPH-CEDAD and ABTS-CE-DAD for the analysis of $\mathrm{DPPH}^{*}$ and $\mathrm{ABTS}^{+\bullet}$ scavenging activities, have been developed to screen radical scavengers in herbal medicines [15-19]. In these on-line methods, antioxidants separated by HPLC and CE will produce their negative peaks in real time detected at $517 \mathrm{~nm}$ for $\mathrm{DPPH}^{\circ}$ or at $414 / 734 \mathrm{~nm}$ for $\mathrm{ABTS}^{+\bullet}$. This avoids the long and tedious process of bioassay-guided fractionation and isolation for structure elucidation in searching antioxidants from complex matrixes. Although the on-line methods have been used to evaluate the antioxidant activity of some herbal medicines, their effectiveness and extensive application need to be further verified.

In this study, an on-line HPLC-UV-ABTS method was developed to screen antioxidants in Dihuang and Shu Dihuang. The chromatographic and active fingerprints could be simultaneously obtained, and antioxidants from the two herbs were compared and identified by HPLCFTMS. The angle cosine method was used to analyze the similarities of the chromatographic and active fingerprints.
Thus, the quality of the herbs could be assessed comprehensively through the proposed research methods.

\section{Methods \\ Materials and reagents}

The information of the Dihuang and Shu Dihuang samples was listed in Additional file 1. Thirteen batches of Dihuang and 13 batches of Shu Dihuang samples were purchased from eleven manufacturers in China. Seven batches of Dihuang samples from the five manufacturers in Hubei province were recorded as S01, S02, S03, S04, S05, S06 and $\mathrm{S} 12$. Four batches of Dihuang samples from the four manufacturers in Anhui province were labeled as S07, S08, S09 and S13, while S10 and S11 were collected from the manufacturers of Guangzhou and Hangzhou, respectively. The thirteen of batches of Shu Dihuang samples from the same manufacturers were labeled as P01-13. Verbascoside was purchased from Chromadex (Irvine, America). ABTS was purchased from TCI (Shanghai, China).

HPLC and MS grade acetonitrile were obtained from Merck Drugs \& Biotechnology (Darmstadt, Germany). Formic acid (FA) was purchased from Aladdin industrial corporation (Shanghai, China). Sodium chloride $(\mathrm{NaCl})$, sodium dihydrogen phosphate $\left(\mathrm{NaH}_{2} \mathrm{PO}_{4}\right)$, potassium chloride $(\mathrm{KCl})$, potassium persulfate $\left(\mathrm{K}_{2} \mathrm{~S}_{2} \mathrm{O}_{8}\right)$, disodium hydrogen phosphate $\left(\mathrm{Na}_{2} \mathrm{HPO}_{4}\right)$, sodium dihydrogen phosphate $\left(\mathrm{NaH}_{2} \mathrm{PO}_{4}\right)$, sodium phosphate $\left(\mathrm{Na}_{3} \mathrm{PO}_{3}\right)$ and acetic acid were analytical reagent grade. The water used was purified from a Millipore water purification system (Millipore, Bedford, MA, USA).

\section{Preparation of sample and standard solutions}

All of the Dihuang and Shu Dihuang samples were dried at $60{ }^{\circ} \mathrm{C}$ under reduced pressure and pulverized to coarse powder. Sample powder (3 g) was extracted twice with $70 \mathrm{~mL}$ methanol for $40 \mathrm{~min}$ by ultra-sonication at room temperature. The extract solutions were filtered and mixed, then evaporated to dryness under vacuum and diluted with $15 \%$ ethanol to $10 \mathrm{~mL}$. After being centrifuged at $10000 \mathrm{rpm}$ for $10 \mathrm{~min}$, an aliquot of $20 \mu \mathrm{L}$ solution was analyzed by HPLC.

Verbascoside $(7.78 \mathrm{mg}$ and $3.81 \mathrm{mg}$ ) was weighed accurately and dissolved in $50 \mathrm{~mL}$ by methanol as the stock solutions. The different concentrations solutions, such as 3.05、6.10、18.7、50.3、99.6 and $155.6 \mu \mathrm{g} / \mathrm{mL}$, were obtained through diluting the stock solutions with methanol. The standard solutions were used to set up the correlative graphs and equations between inhibiting peak areas and the concentrations of verbascoside.

\section{Preparation of ABTS solution}

A stock solution was prepared with ABTS $(0.110 \mathrm{~g})$ dissolved in $100 \mathrm{~mL}$ of PBS solution $(4.1 \mathrm{~g} \mathrm{NaCl}, 0.135 \mathrm{~g}$ $\mathrm{NaH}_{2} \mathrm{PO}_{4}, 0.7 \mathrm{~g} \mathrm{Na}_{2} \mathrm{HPO}_{4}$ and $0.075 \mathrm{~g} \mathrm{KCl}$ in $500 \mathrm{~mL}$ ) 
containing $0.3 \mathrm{mM} \mathrm{K} \mathrm{K}_{2} \mathrm{~S}_{2} \mathrm{O}_{8}$. A $2.0 \mathrm{mM}$ stock solution was diluted using PBS solution containing $10 \%$ methanol to $0.3,0.4,0.5,0.6,0.7$ and $0.8 \mathrm{mM}$, respectively. In online analysis, ABTS solution was freshly prepared and protected from light and cooled in an ice bath.

\section{HPLC-UV-ABTS assay}

The HPLC-UV-ABTS system was reported in our previous publication [20]. The on-line instrumentation consisted of a Waters HPLC for chromatographic fingerprint analysis at $334 \mathrm{~nm}$ or $250 \mathrm{~nm}$ and an Ultimate $3000 \mathrm{UV}$ detector for $\mathrm{ABTS}^{+\cdot}$ scavenging analysis at $734 \mathrm{~nm}$. ABTS solution was delivered with an injection pump of Pickering PCX Della (Pichering Laboratories Inc., USA) at the flow rate of $0.5 \mathrm{~mL} / \mathrm{min}$ after PDA detector, and then the elution was mixed with ABTS solution after PDA detector through a reaction coil with 1.4 $\mathrm{mL}$ volume. The reaction products were determined at $734 \mathrm{~nm}$ by UV detector (Ultimate 3000). All other parts of the HPLC-UV-ABTS system were interconnected using polyether ether ketone (PEEK, $0.5 \mathrm{~mm}$ i.d.) tubes and T-shaped PEEK tubes.

Agilent Extend $\mathrm{C}_{18}$ columns $(250 \mathrm{~mm} \times 4.6 \mathrm{~mm}, 5 \mu \mathrm{m})$ were used for all chromatographic separations. The mobile phase comprised $0.1 \%(\mathrm{v} / \mathrm{v})$ acetic acid (A) and acetonitrile (B). Gradient elution was performed as follows: $2-4 \% \mathrm{~B}$ in $0-10 \mathrm{~min}, 4-15 \% \mathrm{~B}$ in $10-40 \mathrm{~min}, 15-$ $25 \% \mathrm{~B}$ in $40-55 \mathrm{~min}, 25-30 \% \mathrm{~B}$ in $55-60 \mathrm{~min}$ and $30-$ $95 \% \mathrm{~B}$ in $60-65 \mathrm{~min}$. The flow rate was $1.0 \mathrm{~mL} / \mathrm{min}$ and column temperature was maintained at $30^{\circ} \mathrm{C}$. The detection wavelength was set at 334 and $250 \mathrm{~nm}$ for acquiring chromatograms. An aliquot of $20 \mu \mathrm{L}$ sample solution was injected for HPLC-UV-ABTS analysis.

\section{HPLC-FTMS analysis}

Thermo Scientific Orbitrap Fusion Tribrid HPLC-MS system was controlled by Xcalibur software (Version 3.0). The chromatographic conditions were the same as those in the HPLC-UV-ABTS analysis. The mobile phase was split after the DAD detector by a T-tube connected using two PEEK tubes with the same inner i.d. and different length. The split solution with a flow rate of $0.2 \mathrm{~mL} / \mathrm{min}$ finally arrived at the MS detector.

The MS experiments were performed to get an accurate MS and $\mathrm{MS}^{2}$ of the new analogue. The ionization source was operated in the negative ionization modes with the flow rates of the sheath gas and auxiliary gas at 40 and 10 arbitrary unit, respectively, capillary temperature at $320^{\circ} \mathrm{C}$, ion spray source capillary at 2.5 $\mathrm{kV}$, source current at $100 \mu \mathrm{A}$. Six scan events were selected in the MS experiment. Scan event 1 was used for full scan with scan range from 100 to $1000 \mathrm{~m} / \mathrm{z}$ and resolution 60,000 . Scan events $2-6$ were used to produce $\mathrm{MS}^{2}$ through dependent scans selecting the 1th to 5th most intense ions in scan event 1 and resolution 15,000. Collision energy was set at $35 \mathrm{~V}$ using High Energy Collision Dissociation (HCD).

\section{Similarity of the two-dimensional fingerprint}

It is well known that the samples with similar chromatographic fingerprint possess likely similar properties. Therefore, the consistency of herbal medicines can be tested through comparing the similarity between the chromatographic fingerprints of samples and the reference/standard fingerprints.

In this paper, a data analysis method was employed to evaluate the similarity of the two-dimensional fingerprint [21]. The chromatographic and active fingerprints from HPLC-UV-ABTS method were represented mathematically by a vector of their chromatographic peak areas and inhibiting peak areas. Thus, taking the two-dimensional fingerprint as an example, assume that vector $X\left(x_{1}, x_{2}\right.$, $\left.x_{3}, \ldots, x_{\mathrm{n}}\right)\left(|X|=\sqrt{x_{1}{ }^{2}+x_{2}{ }^{2}+x_{3}{ }^{2}+\cdots \cdots \cdot x_{n}{ }^{2}}\right)$ represents the chromatographic or active fingerprint and the other vector $Y\left(y_{1}, \quad y_{2}, \quad y_{3}, \ldots, y_{\mathrm{n}}\right) \quad(|\quad Y|=$ $\left.\sqrt{y_{1}{ }^{2}+y_{2}{ }^{2}+y_{3}{ }^{2}+\cdots \cdots \cdot y_{n}{ }^{2}}\right)$ represents the reference fingerprints, while the vector angle of $X$ and $Y$ is calculated by the formula. The two vectors are more similar when the cosine values are near 1 .

$$
\cos \theta=\frac{X \cdot Y}{|X| \times|Y|}
$$

Where $x_{\mathrm{i}}$ denotes absolute peak area of chromatographic or active fingerprints, and $y_{\mathrm{i}}$ denotes mean area of the peak.

\section{Results}

Optimization of HPLC-UV-ABTS analysis conditions

HPLC-UV-ABTS was developed for the determination of free radical scavengers in the complex matrixes due to a relatively simple and stable instrument system. Methanol/acetonitrile-acid aqueous solution used as mobile phases were compatible with the on-line detection of $\mathrm{ABTS}^{+\bullet}$ inhibition after column or UV detector. Baseline was relatively stable due to the buffer effect of ABTS dissolved in buffer solutions to mobile phases.

The concentrations of the ABTS solution affected directly the sensitivity of the HPLC-UV-ABTS method. In view of this, the different concentrations of ABTS including 0.3, 0.4, 0.5, 0.6, 0.7 and $0.8 \mathrm{mM}$ were investigated. The results showed that the stronger inhibition peaks were produced when the lower concentrations of ABTS were selected, while the higher concentrations induced strong baseline draft. Finally, $0.6 \mathrm{mM}$ ABTS was used to analysis the antioxidants in the two herbs. Additionally, the flow rates at 0.25 and $0.5 \mathrm{~mL} / \mathrm{min}$ for the ABTS solution were 
optimized. The flow rate of $0.5 \mathrm{~mL} / \mathrm{min}$ was finally selected owing to a long time delay from the lower flow rate for $\mathrm{ABTS}^{+\bullet}$ scavenging analysis.

\section{Method validation for HPLC-UV-ABTS}

The analytical methods of the chromatographic and active fingerprints were validated based on retention times, peak areas and $\mathrm{ABTS}^{+\bullet}$ inhibition peak areas. The intraday precisions of HPLC-PDA were $0.086-0.75 \%(n=5)$ for retention times and $0.70-1.46 \%(n=5)$ for peak areas, while the inter-day precisions were $0.10-0.83 \%$ $(n=5)$ for retention times and $1.18-3.37 \%(n=5)$ for peak areas. The intra-day precisions of HPLC-UV-ABTS were within the range of $0.028-0.93 \%(n=5)$ for retention times and $0.069-0.95 \%(n=5)$ for negative peak areas, whereas the inter-day precisions were $1.23-4.73 \%$ $(n=5)$ for retention times and $1.66-5.71 \%(n=5)$ for negative peak areas.

\section{On-line HPLC-UV-ABTS analysis}

The chromatographic and active fingerprints of Dihuang and Shu Dihuang were shown in Fig. 1. Although chromatographic fingerprints of Dihuang and Shu Dihuang were similar, their active fingerprints were different. Fourteen negative peaks were observed in Dihuang (Fig. 1a), while there were 17 inhibition peaks in Shu Dihuang (Fig. 1b). The negative peaks 1, 2, 3, 4, 8, 9, 10 and 16 in Shu Dihuang were stronger than those in Dihuang. In addition, several new negative peaks were found in Shu Dihuang such as 5, 6 and 7, and negative peak 12 in Dihuang disappeared.
In Fig. 1a and b, few peaks were observed from $10 \mathrm{~min}$ to $40 \mathrm{~min}$ in the chromatographic fingerprints of the two herbs detected at $334 \mathrm{~nm}$, while some weak peaks could be found at $250 \mathrm{~nm}$ and these peaks in Shu Dihuang showed obvious ABTS $^{+\bullet}$ inhibition activity, such as peaks 3-9. In view of stronger chromatographic signal at $334 \mathrm{~nm}$ for iridoid glycosides as main components in the two herbs, the chromatographic fingerprints were detected at this wavelength. Furthermore, $\mathrm{ABTS}^{+\bullet}$ scavenging activity was detected usually at $414 \mathrm{~nm}$ and $734 \mathrm{~nm}$, but the wavelength at $734 \mathrm{~nm}$ was selected in order to avoid the interference from the herb extracts at $414 \mathrm{~nm}$.

\section{The activity evaluation of antioxidants in samples}

As shown in Fig. 1, there were 18 antioxidants in the Dihuang and Shu Dihuang samples and their capacities for scavenging $\mathrm{ABTS}^{+\bullet}$ were obviously different. However, it was difficult to evaluate and compare the activity of the antioxidants due to the impossibility of all the standards obtained. Some publications reported that the relative potencies of the antioxidants in complex extracts could be evaluated by the "quantity-effect" equation of a positive control $[22,23]$. Thus the potencies of the antioxidants against free radical could be compared and antioxidant activity of a complex extract could be obtained through calculating the total potency of all the antioxidant components.

In Figs. 1 and 2, verbascoside showed strong ABTS $^{+\cdot}$ scavenging activity in all the Dihuang and Shu Dihuang samples, so it was applied as a positive control. The functional equation of verbascoside $y=2 \mathrm{E}-07 x^{4}-8 \mathrm{E}-$ $05 x^{3}+0.0079 x^{2}+0.5755 x+1.4754\left(R^{2}=1\right)$ was generated

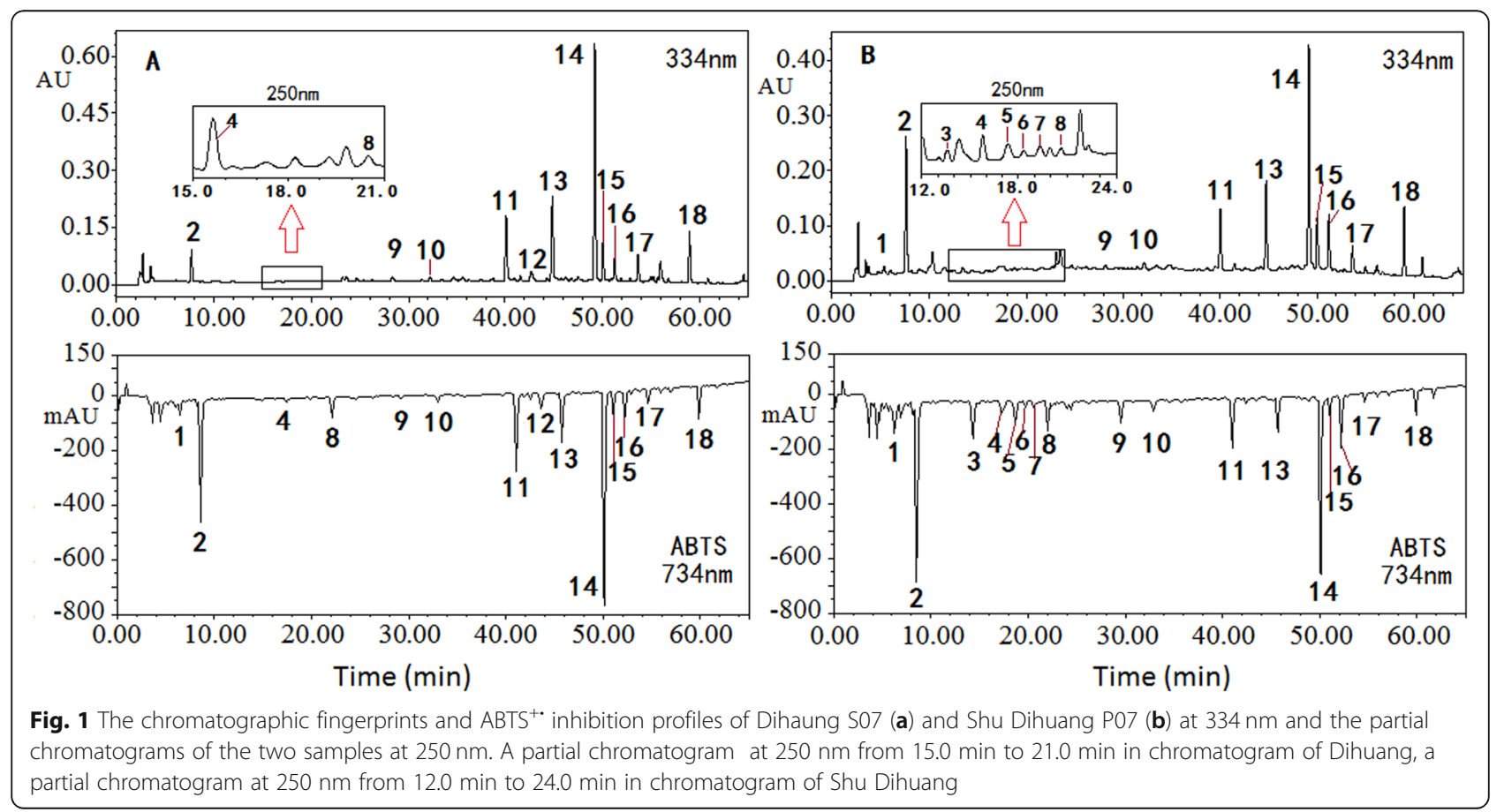



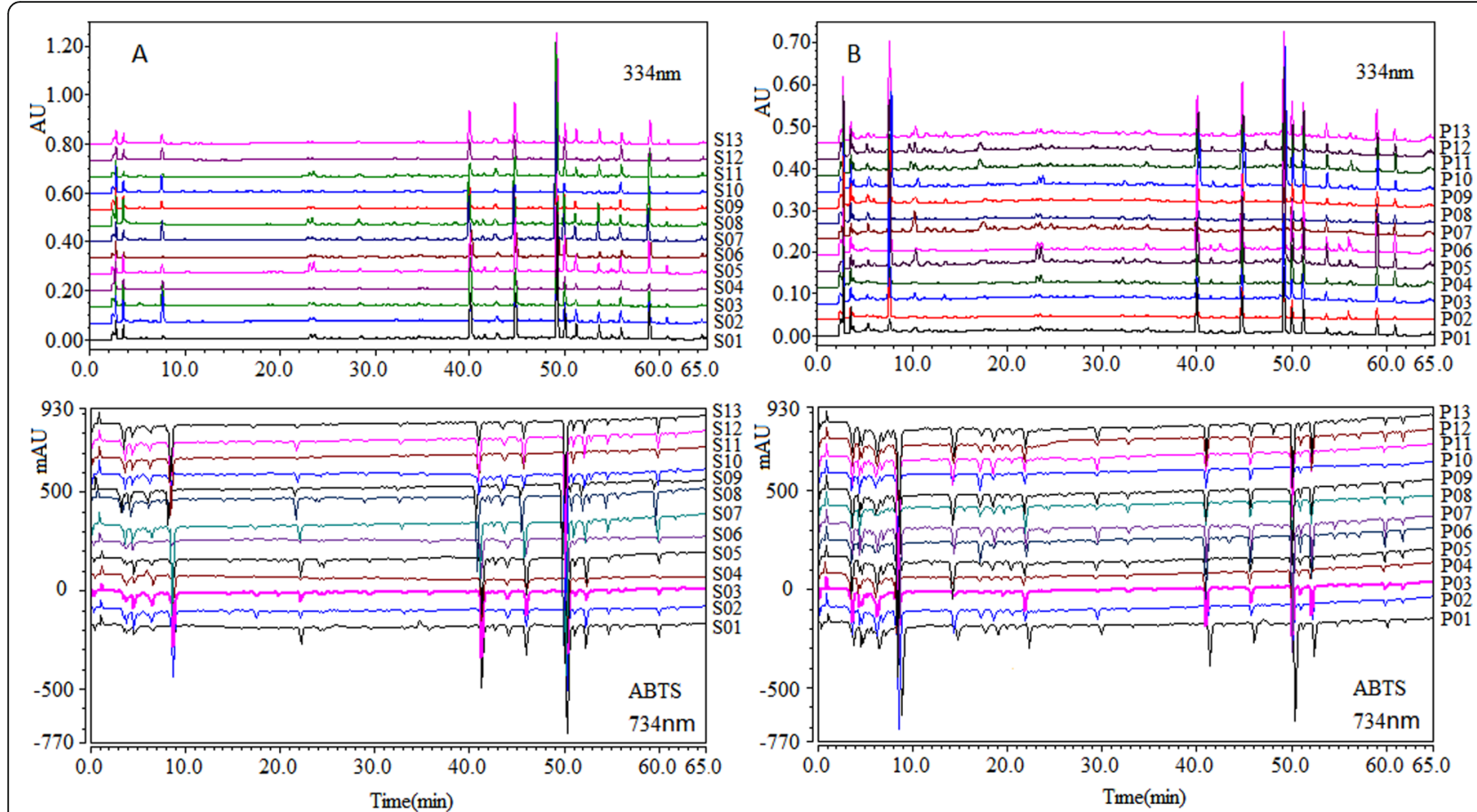

Fig. 2 The chromatographic fingerprints and ABTS ${ }^{+\cdot}$ inhibition profiles of 13 batches of Dihuang and 13 batches of Shu Dihuang samples. a Dihuang, b Shu Dihuang. The chromatographic fingerprints detected at $334 \mathrm{~nm}$. ABTS+• inhibition profiles detected at $734 \mathrm{~nm}$

using negative peak areas (x) and C (y) (Fig. 3) (Additional file 2). If verbascoside $(1 \mu \mathrm{g} / \mathrm{ml})$ was presumed as a potency unit, the potencies of 18 antioxidants in the samples could be calculated by the functional equation (Additional file 3).

The potencies of 18 antioxidants in all of the Dihuang samples and Shu Dihuang samples were shown in Fig. 4a and b. The potencies of the antioxidants in Dihuang and Shu Dihuang were markedly different. In Fig. 4a, peak 2, 11, 12, 13 and 14 in the Dihuang samples showed strong $\mathrm{ABTS}^{+}$inhibition activity, while the antioxidant potencies of peak 1,2 , $3,4,11,13,14$ and 16 in the Shu Dihuang samples were twice to ten times than those in the Dihuang samples (Fig. 4b). In addition, the activity of peak 1 , 2, 3, 4, 8 and 16 in Shu Dihuang increased and this might attributed to the processing action of Dihuang. In our previous study, the HPLC-UV-DPPH method was developed to compare $\mathrm{DPPH}^{\circ}$ scavengers in Dihuang and Shu Dihuang [24], and the results were consistent with the above the results of HPLC-UVABTS analysis.

\section{Main antioxidants in Dihuang and Shu Dihuang}

The eighteen negative peaks in Fig. 1 were identified by HPLC-FTMS and comparison with reference standards and the data in related literatures [12], and the MS data were listed in Table 1 . As shown in Table 1, the most common inhibition peaks were iridoid glycosides, such as peaks 6-18 except for peak 8. In the Dihuang samples, the stronger negative peaks 11, 12, 13, 14 and 16 were identified as echinacoside, isomer of echinacoside, jionoside A1/A2, verbascoside and isoverbascoside, respectively. While peak 1, 4, 8 and 9 showing stronger activity in the Shu Dihuang samples were identified as glutinoside, geniposidic acid, mussarnosidic acid, and syringic acid-4-O- $\alpha-L$-rhamnoside, respectively. Peaks 2 and 3 needed to be further identified.

Iridoid glycosides are main ingredients in Dihuang and Shu Dihuang, and verbascoside exhibiting stronger antioxidant activity is used to control quality of the two herbs in Ch. P. However, the activity of the other iridoid glycosides can't be ignored. The contributions of 18 antioxidants in the Dihuang and Shu Dihuang samples to the total activity were shown in Fig. 5 (Additional file 4). In Fig. 5a, peaks 2, 11 and 14 in the Dihuang samples exhibited their high contributions, and the total contribution percentage of the three main active components in the Dihuang was $39.2-58.1 \%$. Peaks 1, 2, 3, 11, 14 and 16 in the Shu Dihuang samples showed their high contributions (Fig. 5b), and the total contribution percentage of peak 2, 11 and 14 was $55.9-69.4 \%$. In addition, the contributions of peak 1 , 2, 3, 4, 8 and 16 in the Shu Dihuang samples were twice to twenty times than those in the Di Huang samples, while 


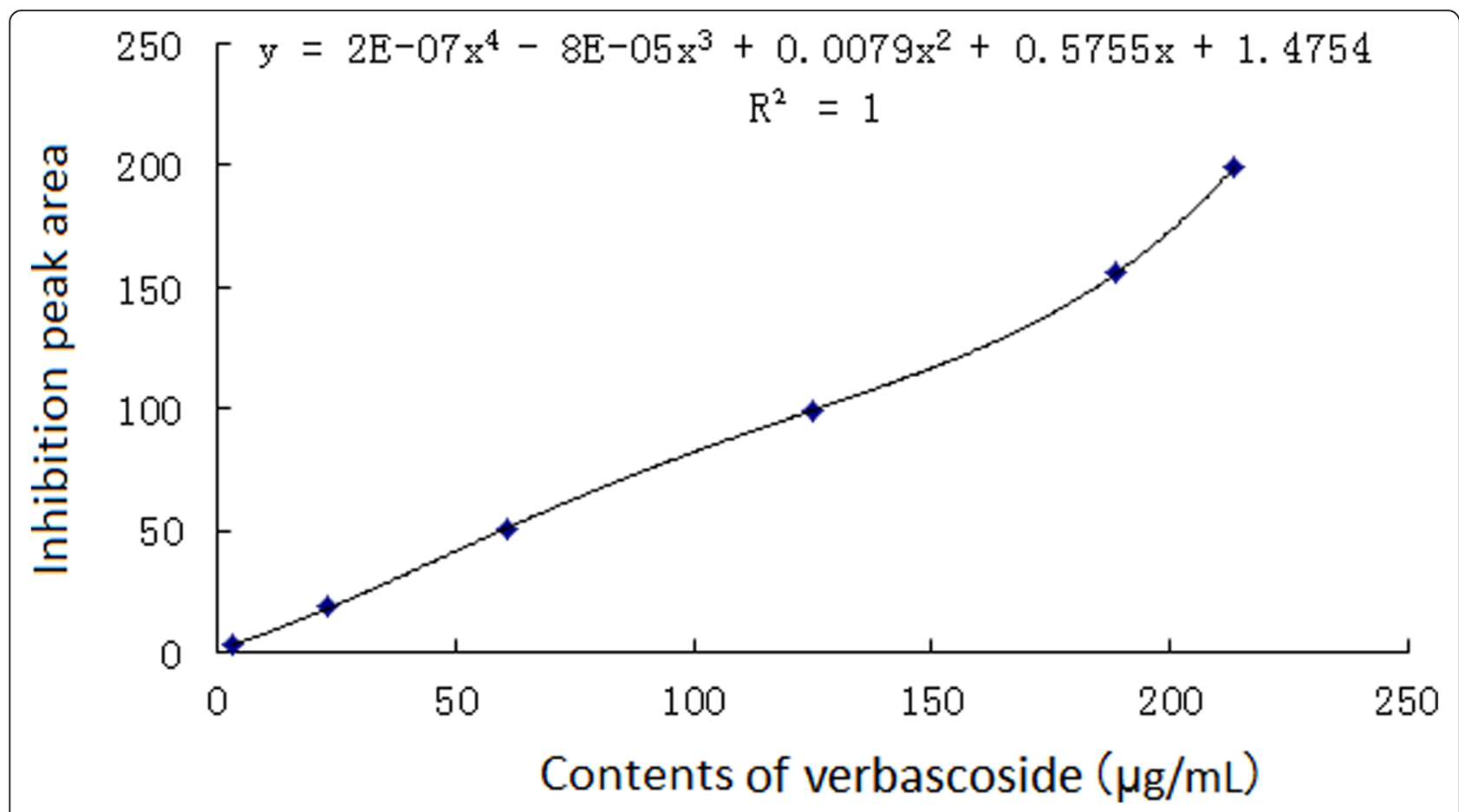

Fig. 3 The correlative graphs and equations between the negative peak areas and concentrations for verbascoside against ABTS ${ }^{+}$

the contributions of peak 11,13 and 14 in the Shu Dihuang samples decreased. The results indicated that the antioxidant activity of Dihuang might be changed after being processed.

\section{Similarity for Dihuang and Shu Dihuang samples}

The chromatographic and active fingerprints of the thirteen batches of the Dihuang and Shu Dihuang samples from the different manufacturers were displayed in Fig. 2. As shown in Fig. 2a and b, the chromatographic and active fingerprints among the different samples were similar. In order to evaluate objectively the quality of the samples, the angle cosine method was employed to analyze the similarities of the two fingerprints. The angle cosine values of each chromatographic and active fingerprint to their respective reference chromatogram were calculated and listed in Table 2. The raw data were shown in Additional file 5 and Additional file 6. In Table 2, the different common peaks were used to evaluate the

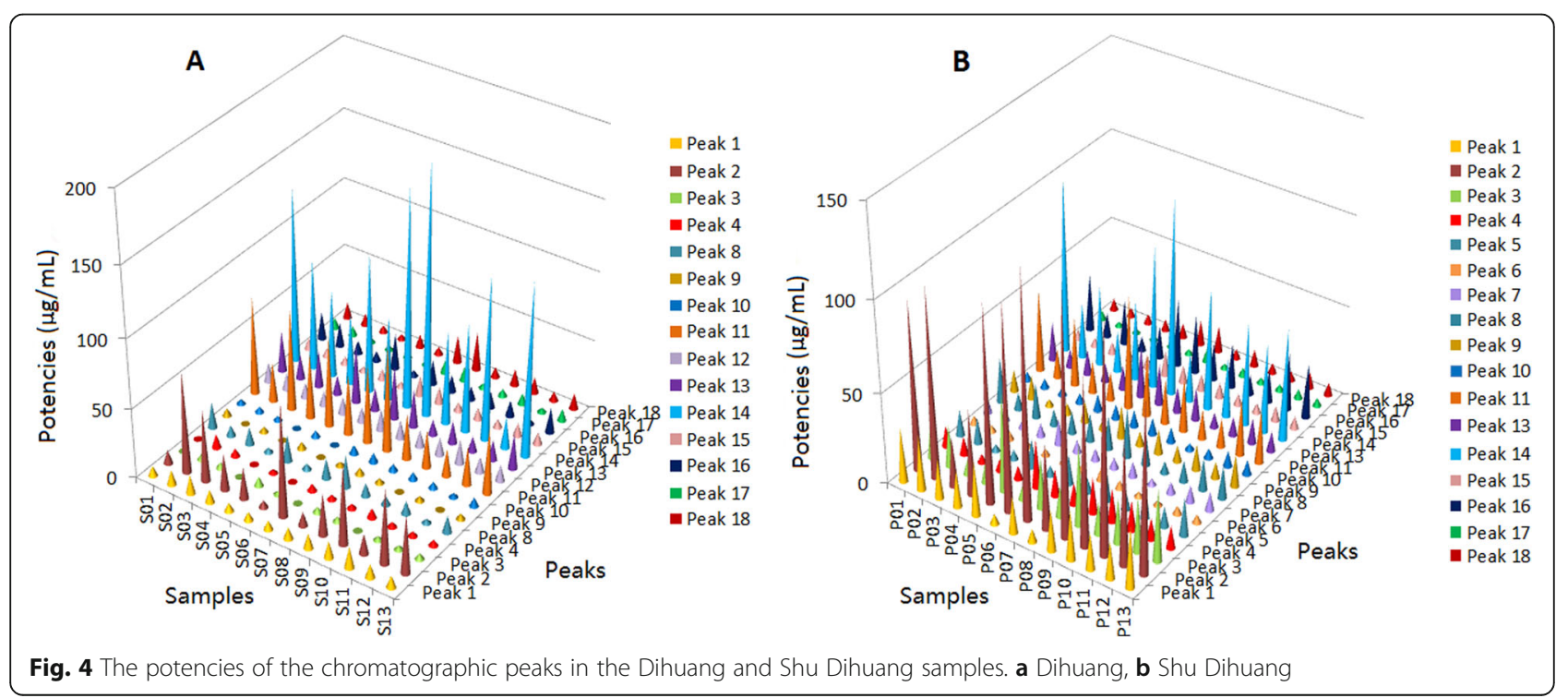


Table 1 Identification of active components in Dihuang and Shu Dihuang sample by HPLC-FTMS

\begin{tabular}{llllll}
\hline Peak & $R T$ & {$[\mathrm{M}-\mathrm{H}]-$} & $\mathrm{Chemical}$ formula & $\mathrm{MS}^{2}$ & Identification \\
\hline 1 & 6.22 & 397.0238 & $\mathrm{C}_{15} \mathrm{H}_{23} \mathrm{O}_{10} \mathrm{Cl}$ & $379.1123,276.9031,252.2112$ & glutinoside \\
2 & 8.55 & 495.1254 & $\mathrm{C}_{26} \mathrm{H}_{23} \mathrm{O}_{10}$ & $477.1181,379.1190,217.0684,199.0581$ & unknown \\
3 & 14.37 & 731.2100 & $\mathrm{C}_{29} \mathrm{H}_{33} \mathrm{O}_{14} \mathrm{~N}_{9}$ & $505.1492,323.0935,263.0734,221.0633$ & unknown \\
4 & 17.25 & 373.1073 & $\mathrm{C}_{16} \mathrm{H}_{22} \mathrm{O}_{10}$ & $283.0792,211.0580,167.0687,123.0431$ & geniposidic acid \\
5 & 18.61 & 375.1246 & $\mathrm{C}_{16} \mathrm{H}_{24} \mathrm{O}_{10}$ & $315.1030,255.0831,213.0735,169.0843,125.0586$ & 8-epiloganic acid \\
6 & 19.60 & 389.1168 & $\mathrm{C}_{17} \mathrm{H}_{26} \mathrm{O}_{10}$ & $183.0634,165.0531,139.0377$ & loganin \\
7 & 20.55 & 461.1592 & $\mathrm{C}_{20} \mathrm{H}_{30} \mathrm{O}_{12}$ & $315.1039,297.0937,161.0429,135.0429$ & decaffeoylacteoside \\
8 & 21.94 & 375.1246 & $\mathrm{C}_{16} \mathrm{H}_{24} \mathrm{O}_{10}$ & $315.1021,213.0738,169.0843,151.0740$ & mussarnosidic acid \\
9 & 29.57 & 343.0262 & $\mathrm{C}_{15} \mathrm{H}_{20} \mathrm{O}_{9}$ & $299.1118,284.0858,197.0424,182.0192$ & syringic acid-4-O-a-L-rhamnoside \\
10 & 32.87 & 475.1747 & $\mathrm{C}_{21} \mathrm{H}_{32} \mathrm{O}_{12}$ & $329.1194,311.1093,143.0583,161.0429$ & darendoside B \\
11 & 41.02 & 785.2394 & $\mathrm{C}_{35} \mathrm{H}_{45} \mathrm{O}_{20}$ & $623.2108,461.1603,161.0218$ & echinacoside \\
12 & 43.60 & 785.2394 & $\mathrm{C}_{35} \mathrm{H}_{45} \mathrm{O}_{20}$ & $623.2108,461.1603,161.0218$ & isomer of echinacoside \\
13 & 45.74 & 799.2570 & $\mathrm{C}_{36} \mathrm{H}_{48} \mathrm{O}_{20}$ & $623.2102,605.1965,461.1597,315.1040$ & jionoside A1/A2 \\
14 & 50.14 & 623.1865 & $\mathrm{C}_{29} \mathrm{H}_{36} \mathrm{O}_{15}$ & $477.1354,461.1599,443.1508,315.1039,179.0321,161.0218$ & verbascoside \\
15 & 50.98 & 813.2729 & $\mathrm{C}_{37} \mathrm{H}_{50} \mathrm{O}_{20}$ & $637.2254,491.1701,193.0477,175.0373$ & jionoside B1/B2 \\
16 & 52.17 & 623.1865 & $\mathrm{C}_{29} \mathrm{H}_{36} \mathrm{O}_{15}$ & $461.1600,315.1040,251.0525,179.0322,161.0218$ & isoverbascoside \\
17 & 54.61 & 637.2048 & $\mathrm{C}_{30} \mathrm{H}_{38} \mathrm{O}_{15}$ & $491.1492,475.1773,461.4599,443.1497$ & jionoside D/leucosceptoside A \\
18 & 59.89 & 651.2176 & $\mathrm{C}_{31} \mathrm{H}_{40} \mathrm{O}_{15}$ & 505.1632475 .1754193 .0477 & martynoside/isomer
\end{tabular}

similarities of the two fingerprints. The peak 12 was not detected in the Shu Dihuang samples, so 8 common peaks were selected in the similarity evaluation of the chromatographic fingerprints. Fifteen and seventeen common peaks were used to analyze the similarity of the active fingerprints of the Dihuang and Shu Dihuang samples, respectively.

As shown in Table 2, the chromatographic and active fingerprints of the samples represented their differences to some extent. The similarity values of S03 and $\mathrm{S} 12$ in the two fingerprints were lower than those of the other samples, and their similarity values in the active fingerprints were less than 0.9. Moreover, the similarity value of P03 in the chromatographic fingerprints was lower than that of the other samples and less than 0.9 in the active fingerprints. Although the similarity values of the two fingerprints were differences, the quality of the Dihuang and the Shu Dihuang samples were basically consistent except for the several samples.

\section{Discussion}

In this study, the HPLC-UV-ABTS method was developed to evaluate the main antioxidants and quality of

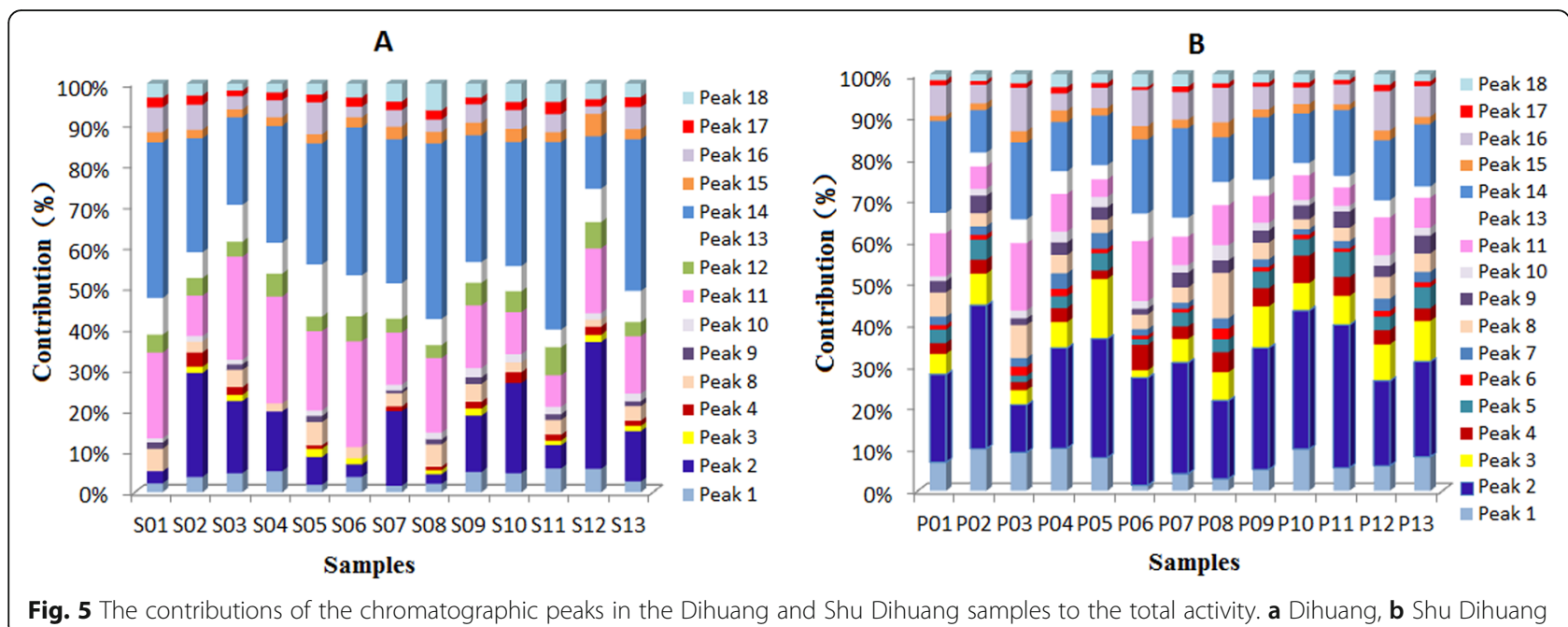

Fig. 5 The contributions of the chromatographic peaks in the Dihuang and Shu Dihuang samples to the total activity. a Dihuang, b Shu Dihuang 
Table 2 Similarity of Dihuang and Shu Dihuang samples

\begin{tabular}{|c|c|c|c|c|c|}
\hline \multirow[t]{2}{*}{ Samples } & \multicolumn{2}{|l|}{ Dihuang } & \multirow[t]{2}{*}{ Samples } & \multicolumn{2}{|l|}{ Shu Dihuang } \\
\hline & HPLC (9 common peaks) & ABTS (15 common peaks) & & HPLC (8 common peaks) & ABTS (17 common peaks) \\
\hline S01 & 0.994 & 0.982 & P01 & 0.991 & 0.969 \\
\hline S02 & 0.954 & 0.957 & P02 & 0.964 & 0.959 \\
\hline S03 & 0.903 & 0.898 & P03 & 0.902 & 0.854 \\
\hline S04 & 0.952 & 0.958 & P04 & 0.952 & 0.980 \\
\hline S05 & 0.988 & 0.978 & P05 & 0.968 & 0.961 \\
\hline S06 & 0.975 & 0.969 & P06 & 0.976 & 0.955 \\
\hline S07 & 0.994 & 0.974 & P07 & 0.984 & 0.984 \\
\hline S08 & 0.989 & 0.974 & P08 & 0.932 & 0.944 \\
\hline S09 & 0.992 & 0.988 & P09 & 0.987 & 0.991 \\
\hline S10 & 0.991 & 0.934 & P10 & 0.983 & 0.966 \\
\hline S11 & 0.941 & 0.946 & P11 & 0.946 & 0.975 \\
\hline S12 & 0.840 & 0.716 & P12 & 0.974 & 0.984 \\
\hline $\mathrm{S} 13$ & 0.994 & 0.993 & P13 & 0.993 & 0.989 \\
\hline
\end{tabular}

the two herbs. Sensitivity of the on-line method correlated mainly concentration and flow rate of ABTS solution. Lower concentrations of ABTS solution were used, and stronger inhibition peaks in the samples could be obtained. Considering strong baseline drift induced by the higher concentrations of ABTS solution, $0.6 \mathrm{mM}$ within the range of $0.3-0.8 \mathrm{mM}$ was selected for the on-line analysis. Moreover, flow rate of ABTS solution affected directly the reaction times of the compounds and $\mathrm{ABTS}^{+\cdot}$ when volume of reaction coil $(1.4 \mathrm{~mL})$ and flow rate of HPLC elution were stable. Flow rate of $0.5 \mathrm{~mL} / \mathrm{min}$ for ABTS solution was applied due to a long time delay and lower resolution of the active fingerprints from the lower flow rates. Under the above analysis conditions, the method validation of HPLC-UV-ABTS was investigated through the intra-day and inter-day precisions of the chromatographic peaks, the negative peaks and retention times. The results indicated that the on-line method was stable and reproducible.

The chromatographic fingerprints of all the samples were detected at $334 \mathrm{~nm}$, while some minor peaks were found in the chromatographic fingerprints of Shu Dihuang at $250 \mathrm{~nm}$ and showed their $\mathrm{ABTS}^{+\cdot}$ scavenging activity. These minor peaks are hardly focused on owing to their weak signals in the chromatographic fingerprints. Chromatographic fingerprint mainly showing characteristics of strong peaks gave too much weight to major peaks than to minor peaks. In view of this, the similarities of the chromatographic and active fingerprints were evaluated and compared by the vector angle cosine method. Similarity values of several samples were less than 0.9. This might imply their different quality with the other samples. Thus, the combination method of the chromatographic and active fingerprints could evaluate objectively the quality of herbal medicines.

In order to assess and compare the activity of the components in the Dihuang and Shu Dihuang samples, verbascoside was used as a positive control to obtain the relative activity of the $\mathrm{ABTS}^{+}{ }^{-}$inhibition peaks. The results found that the active components in the two herbs were markedly different. Contents of some components in Dihuang were changed after being processed so as to induce their different activity in the two herbs. This might imply the different therapeutic effects of the two herbs in clinical to some extent. Thus, the "quantity-effect" research method displayed its important action in the quality evaluation and identification of the active compounds of herbal medicines.

\section{Conclusion}

In this study, the on-line HPLC-UV-ABTS method and a "quantity-effect" research idea were developed to evaluate the quality and antioxidant activity of the Dihuang and Shu Dihuang samples from the different manufacturers. The results revealed that the antioxidants in the Shu Dihuang samples were obviously different from those in the Dihuang samples owing to the contents of some components having changed after Dihuang was processed. Iridoid glycosides were the main antioxidants and their contributions to the total activity were higher, while the new produced ingredients in Shu Dihuang displaying significant $\mathrm{ABTS}^{+\bullet}$ inhibition activity could not be ignored. In short, the HPLC-UV-ABTS 
method was simple, rapid and reliable and could be applied widely to screen the antioxidants and evaluate the antioxidant activity of the complex extracts. The combination method of the chromatographic and active fingerprints could evaluate integratedly the active components of the complex matrixes and might be valuable and meaningful for improving the quality control of herbal medicines.

\section{Supplementary information}

Supplementary information accompanies this paper at https://doi.org/10 1186/s12906-019-2798-8

Additional file 1. A table for manufacturer source of the Dihuang and Shu Dihuang samples.

Additional file 2. The raw data for the functional equation of verbascoside as a positive control, negative peak area (x), concentration (y).

Additional file 3. The peak areas and potencies of the negative peaks in the thirteen batches of Dihuang and Shu Dihuang samples. Potency of each negative peak calculated by the functional equation of verbascoside.

Additional file 4. The raw data of Figs. 4 and 5. The potencies of 18 negative peaks in 13 batches of Dihuang and Shu Dihuang samples.

Additional file 5. The potencies of the negative peaks in the active fingerprints and their similarity evaluation. The similarity values of the active fingerprints of 13 batches of Dihuang and Shu Dihuang samples calculated by the angle cosine formula.

Additional file $\mathbf{6}$. The peak areas of the chromatoghraphic fingerprints and their similarity evaluation. The similarity values of the chromatographic fingerprints of 13 batches of Dihuang and Shu Dihuang samples calculated by the angle cosine formula.

\section{Abbreviations}

ABTS: 2, 2'-azino-bis(3-ethylbenzothiazoline-6-sulfonic acid); BCD: Biological chemistry detection; Ch. P.: Chinese Pharmacopoeia; DPPH: 2,2-diphenyl-1picrylhydrazyl hydrate; HPLC: high performance liquid chromatographic; HPLC-FTMS: HPLC-fourier-transform mass spectrometry; HPLC-PDA: HPLCphoto-diode array; HPLC-UV-ABTS: high performance liquid chromatographic-ultraviolet detection-ABTS

\section{Acknowledgements}

We are pleased to thank Thermo Fisher Scientific for technical support.

\section{Authors' contributions}

$\mathrm{HL}$ and JF contributed equally to this work. HL and JF conceived and designed the study. HS performed the HPLC-FTMS analysis experiment and collected the data. XZ analyzed the data and supervised the work. XD collected samples from different manufactories and wrote the manuscript. JL involved in the initial experimental design, and provided a great help and careful guide for the twice modification of the manuscript including the data processing analysis and grammar part. All authors read and approved the final manuscript.

\section{Funding}

This work was financially supported by Natural Science Foundation of China (Grant no. 81403093), Science Foundation of Hubei Food and Drug Administration (Grant no. $2016010+02$ ) and Natural Science Foundation of Hubei Province (No. 2015CFB493). The on-line HPLC-UV-ABTS method derived from the study of Natural Science Foundation of China. This work provided research data to finish Science Foundation of Hubei Food and Drug Administration. The design and idea of this study were based on Natural Science Foundation of Hubei Province.

\section{Availability of data and materials}

The datasets used and/or analyzed during the current study are available from the corresponding author on reasonable request.

\section{Ethics approval and consent to participate}

Not applicable.

\section{Consent for publication}

Not applicable.

\section{Competing interests}

The authors declare that they have no competing interests.

\section{Author details}

${ }^{1}$ Hubei Institute for Drug Control, Wuhan 430075, Hubei, China. ${ }^{2}$ School of Chemistry, Chemical Engineering and Life Sciences, Wuhan University of Technology, Wuhan 430070, Hubei, China. ${ }^{3}$ MOE Key Laboratory of Chinese Medicine Resource and Compound Prescription, Hubei University of Chinese Medicine, Wuhan 430065, Hubei, China.

Received: 12 November 2019 Accepted: 17 December 2019 Fective

\section{References}

1. Xu ZJ, Shu S, Li ZJ, Liu YM, Zhang RY, Zhang Y. Liuwei Dihuang pill treats diabetic nephropathy in rats by inhibiting of TGF-B/SMADS, MAPK, and NF$\mathrm{kB}$ and upregulating expression of cytoglobin in renal tissues. Medicine (Baltimore). 2017:96:e5879.

2. Zhao M, Tao J, Qian D, Liu P, Shang E, Jiang S, et al. Simultaneous determination of loganin, morroniside, catalpol andacteoside in normal and chronic kidney disease rat plasma by UPLC-MS for investigating the pharmacokinetics of Rehmannia glutinosa and Cornus officinalis Sieb drug pair extract. J Chromatogr B. 2016;1009:122-9.

3. Yuhong Y, Qian L, Yu L, Yingqiang Z, Yanfen L, Shujing Y, et al. An n-of-1 trial service in clinical practice: testing the effectiveness of Liuwei Dihuang Decoction for kidney-yin deficiency syndrome. Evid Based Complement Alternat Med. 2013:2013:827915.

4. Zhu H, Wang Y, Liu Z, Wang J, Wan D, Feng S, et al. Antidiabetic and antioxidant effects of catalpol extracted from Rehmannia glutinosa (Di Huang) on rat diabetes induced by streptozotocin and high-fat, high-sugar feed. Chin Med. 2016;11:25

5. Li ZF, He CL, Wang Y, Li MJ, Dai YJ, Wang T, et al. Enhancement of trichothecene mycotoxins of Fusarium oxysporum by ferulic acid aggravates oxidative damage in Rehmannia glutinosa Libosch. Sci Rep. 2016; 6:33962

6. Yu HH, Seo SJ, Kim YH, Lee HY, Park RK, So HS, et al. Protective effect of Rehmannia glutinosa on the cisplatin-induced damage of HEl-OC1 auditory cells through scavenging free radicals. J Ethnopharmacol. 2006:107(3):383-8.

7. Chao JC-J, Chiang SW, Wang CC, Tsai YH, Wu MS. Hot water-extracted Lycium barbarum and Rehmannia glutinosa inhibit proliferation and induce apoptosis of hepatocellular carcinoma cells. World J Gastroenterol. 2006;12: 4478-84.

8. Liu CL, Cheng L, Ko CH, Wong CW, Cheng WH, Cheung DW, et al. Bioassayguide disolation of anti-inflammatory components from the root of Rehmannia glutinosa and its underlying mechanism via inhibition of iNOS pathway. J Ethnopharmacol. 2012;143:867-75.

9. Huang Y, Qin T, Huang Y, Liu Z, Bo R, Hu Y, et al. Rehmannia glutinosa polysaccharide liposome as a novel strategy for stimulating an efficient immune response and their effects on dendritic cells. Int J Nanomedicine. 2016;11:6795-808

10. Feng WS, Li M, Zheng XK, Zhang N, Song K, Wang JC, et al. Two new ionone glycosides from the roots of Rehmannia glutinosa Libosch. Nat Prod Res. 2015;29:59-63.

11. Lee SY, Kim JS, Chio RJ, Kim YS, Lee JH, Kang SS. A new polyoxygenated triterpene and two new aeginetic acid quinovosides from the roots of Rehmannia glutinosa. Chem Pharm Bull. 2011;59:742-6.

12. Song Q, Zhao Y, Zhang N, Zhang Q, Liu Y, Li J, et al. Establishment of HPLC fingerprint of Rehmanniae radix and its HPLC-ESI-MS analysis. Chin Tradit Herbal Drugs. 2016;47(23):4247-52.

13. Chinese Pharmacopoeia Commission. Pharmacopoeia of the People's Republic of China, vol. 1; 2015. p. 125-6. 
14. Martínez-Cayuela M. Oxygen free radicals and human disease. Biochimie. 1995:77:147-61.

15. Lee KJ, Oh YC, Cho WK, Ma JY. Antioxidant and anti-inflammatory activity determination of one hundred kinds of pure chemical compounds using offline and online screening HPLC assay. Evid Based Complement Alternat Med. 2015;2015:165457.

16. Karaçelik AA, Küçük M, Iskefiyeli Z, Aydemir S, De Smet S, Miserez B, et al. Antioxidant components of Viburnum opulus $L$. determined by on-line HPLC-UV-ABTS radical scavenging and LC-UV-ESI-MS methods. Food Chem. 2015;175:106-14.

17. Niederländer HAG, van Beek TA, Bartasiute A, Koleva II. Antioxidant activity assays on-line with liquid chromatography. J Chromatogr A. 2008;1210:12134.

18. Liu J, Tian J, Li J, Azietaku JT, Zhang B, Gao X, Chang Y. In-capillary DPPHcapillary electrophoresis-the diode array detector combined with reversedelectrode polarity stacking mode for screening and quantifying major antioxidants in Cuscuta chinensis lam. Electrophoresis. 2016;37:1632-9.

19. Ma H, Li J, An M, Gao XM, Chang YX. A powerful on line ABTS-CE-DAD method to screen and quantify major antioxidants for quality control of Shuxuening Injection. Sci Rep. 2018;8:5441.

20. Peng WB, Zeng QH, Li DP, Ding TM, Tan JL, Ding XP. Multiple on-line HPLC coupled with biochemical detection methods to evaluate bioactive compounds in Danshen injection. Biomed Chromatogr. 2016;30:1854-60.

21. Wang $L X$, Xiao HB, Liang XM, Bi KS. Vectorial angle method for evaluating the similarity between two chromatographic fingerprints of Chinese herb. Yao Xue Xue Bao. 2002;37(9):713-7.

22. Ding XP, Qi J, Chang YX, Mu LL, Zhu DN, Yu BY. Quality control of flavonoids in Ginkgo biloba leaves by high-performance liquid chromatography with diode array detection and on-line radical scavenging activity detection. J Chromatogr A. 2009;1216:2204-10.

23. Ding XP, Wang XT, Chen LL, Qi J, Xu T, Yu BY. Quality and antioxidant activity detection of Crataegus leaves using high-performance liquid chromatography with diode array detector coupled to chemiluminescence detection. Food Chem. 2010;120:929-33.

24. Shen HD, Fang JJ, Guo PC, Ding TM, Liu JF, Ding XP. Study of anti-oxidants of Rehmanniae Radix and Rehmannia Radix Praeparata by HPLC-UV-DPPH method. Chin Tradit Herbal Drugs. 2018:49:582-8.

\section{Publisher's Note}

Springer Nature remains neutral with regard to jurisdictional claims in published maps and institutional affiliations.

Ready to submit your research? Choose BMC and benefit from:

- fast, convenient online submission

- thorough peer review by experienced researchers in your field

- rapid publication on acceptance

- support for research data, including large and complex data types

- gold Open Access which fosters wider collaboration and increased citations

- maximum visibility for your research: over $100 \mathrm{M}$ website views per year

At $\mathrm{BMC}$, research is always in progress.

Learn more biomedcentral.com/submissions 